Nature of Study Vehicular accidents are the major cause of head injury world over due to persistent increase in fatal vehicular accident in least 25 years. There has been considerable increase in death due to head injury in Chandigarh zone of North West India. Very little information regarding the epidemiology of cranio-intracranial injuries is available from North West India in general and Chandigarh zone in particular. Present study is an attempt to establish the demographic profile, made and extent of head injury among those who died in Postgraduate Institute of Medical Education and Research, Chandigarh, India.

Material and Method Present study is an analysis of the autopsy records of 2982 subjects who were admitted and died because of fatal vehicular accidents at Postgraduate Institute of Medical Education and Research, Chandigarh (India) between 1 February 1987-31 January 2012. Postmortem examination on these subjects was conducted by the department of Forensic Medicine, PGIMER, Chandigarh. Information regarding the age, gender, mode of injury, time of injury, type of vehicle involved and treatment given including surgery were recorded from the postmortem and hospital records which also has further confirmation from the reports of victims relatives, friends and police.

Results Fatal vehicular accidents constituted (50.3\%) major cause of unnatural deaths Postgraduate Institute of Medical Education and Research, Chandigarh (India) and its incidence increased from 50.6\% to $54.7 \%$ between $1987-1992$ then decreased to $45.3 \%$ in next decade and then again increased to $50.2 \%$ in $2002-2012$ ( $p<0.01$ ). In $18 \%$ victims blood alcohol level was above the permissible limit Productive age group that is, $16-45$ years $(70 \%)$ with male preponderance $(88.96 \%)$ was observed throughout the study period.

Two wheelers occupants (motorcycles, scooters) were the major victims of fatal vehicular accidents (33.3\%) followed by pedestrians (23\%) bicyclist (22\%) and four wheelers occupants $(21.7 \%)$. Among the two wheelers victims (40.54\%) were without helmets and $(22.43 \%)$ were with ill-fitted helmets.

$58 \%$ victims died within $24 \mathrm{~h}$ of accidents and head injury was the most common cause of death (79.64\%). Temporal bone was most commonly involved (69.09\%) and occipital bone the least (9.98\%). Subdural haematoma (26.95\%) was common followed by extradural haematoma (23.7\%) intracranial haemorrhage $(5.43 \%)$.

In $43.9 \%$ subjects various combination of intra-cranial haemorrhage was seen. Injury to brain tissues was seen in $78.27 \%$ of instances and most commonly affected were the temporal lobes $(56.4 \%)$ other associated injuries were fracture of long bones $(33.47 \%)$ ribs $(21.67 \%)$, vertebrae $(5.47 \%)$, and sternum $(3.45 \%)$. In $23.74 \%$ instances thoracoabdominal injuries were also present.

\title{
PATTERN OF CRANIO-INTRACRANIAL INJURIES IN FATAL VEHICULAR ACCIDENTS IN CHANDIGARH ZONE OF NORTH -WEST INDIA-A 25 YEARS AUTOPSY EXPERIENCE FROM A TERTIARY CARE HOSPITAL OF INDIA
}

doi:10.1136/injuryprev-2012-040590u.39

D Singh. Postgraduation Institute of Medical Education \& Research

\section{Objectives}

- To analyse the epidemiological factors involved in fatal vehicular accidents as observed in hospital setting

- To analyse the pattern of cranio-intracranial injuries in fatal vehicular accidents

- To provide a base line data to policy makers to help in planning the preventive and curative measures pertaining to human habitations highways, industries and also to equip the health care institution accordingly. 